\title{
CALCIFICAÇÕES CEREBRAIS DIFUSAS EM PACIENTE COM HIPOPARATIREOIDISMO SECUNDÁRIO
}

\section{Antonio Marcos da Silva Catharino, fernanda Martins Coelno Catharino, Marco Orsini, Marcelo Namen}

Trabalho realizado no Hospital Geral de Nova Iguaçu; na Policlínica Geral de Nova Iguaçu; Universidade Iguaçu - UNIG; e na Universidade Federal do Estado do Rio de Janeiro - UNIRIO

LMO, sexo feminino, 58 anos, procurou o ambulatório com intuito de realizar acompanhamento neurológico de seqüela de doença cerebrovascular isquêmica ocorrida na vigência de pico hipertensivo, há três anos, que deu origem a crises epilépticas tônico-clônicas generalizadas tratadas, desde então com medicação anticonvulsivante.

O exame neurológico evidenciava hemiparesia leve à esquerda, apraxia e discreto déficit cognitivo caracterizado por dificuldade de concentração, atenção e déficit de memória. Segundo informação de familiares, tais alterações tiveram início após a injúria vascular.

A tomografia computadorizada de crânio revelava área hipodensa em região parietal direita, compatível com seqüela de injúria vascular isquêmica, e calcificações difusas em substância branca periventricular, núcleos da base e cerebelo (Figuras I, 2 e 3).

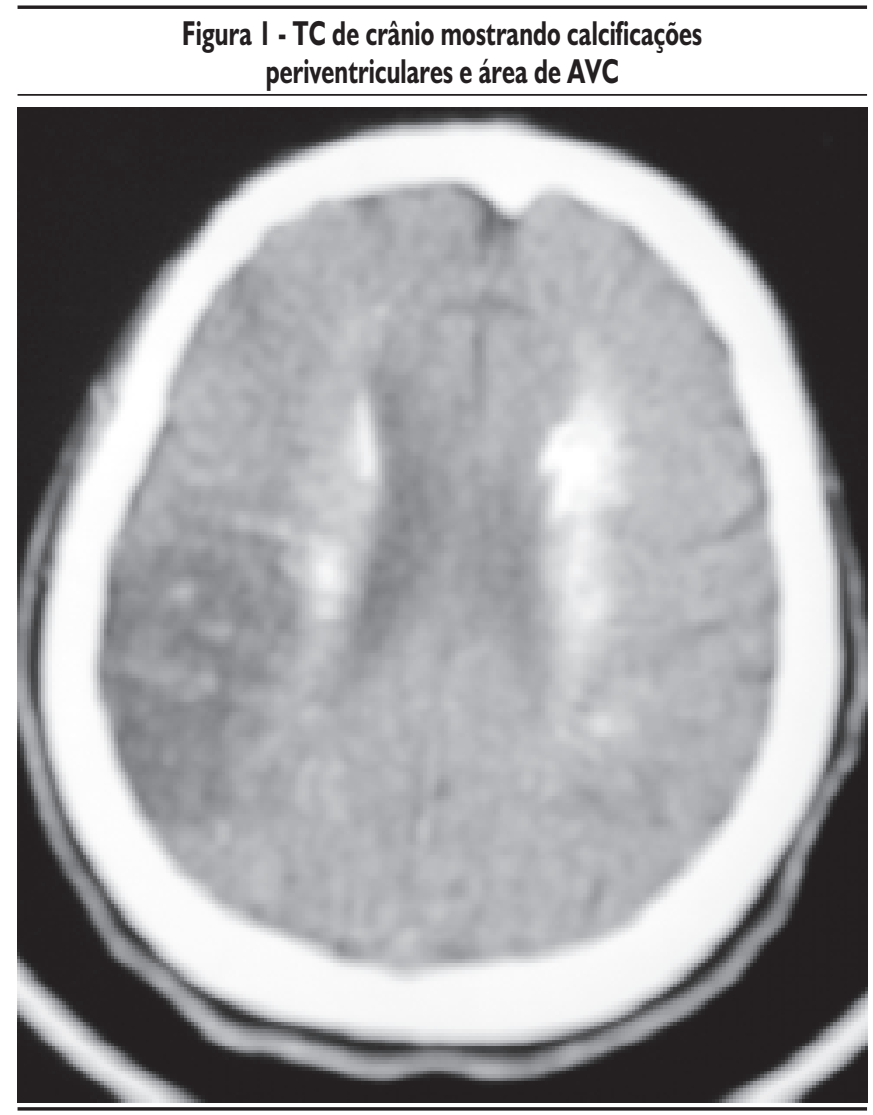

A paciente apresentava, como antecedentes patológicos, hipertensão arterial sistêmica, em acompanhamento cardiológico desde o insulto cerebrovascular. Diabetes mellitus tipo II e hipoparatireoidismo secundário a tireoidectomia total realizada há 30 anos, desde então se encontrava em acompanhamento endocrinológico por conta destas doenças. Os exames laboratoriais revelavam discreta hiperglicemia $(123 \mathrm{mg} / \mathrm{dl})$ e nível sérico de cálcio dentro dos limites da normalidade.

A identificação de calcificações intracranianas é freqüente em exames de imagem!. Muitas alterações metabólicas podem estar relacionadas a este quadro, dentre elas as disfunções da paratireóide $^{2}$. O hipoparatireoidismo pode ser secundário a tireoidectomia total ou parcial e está entre as causas de calcificações cerebrais $^{3}$. Os achados tomográficos desta condição podem ser

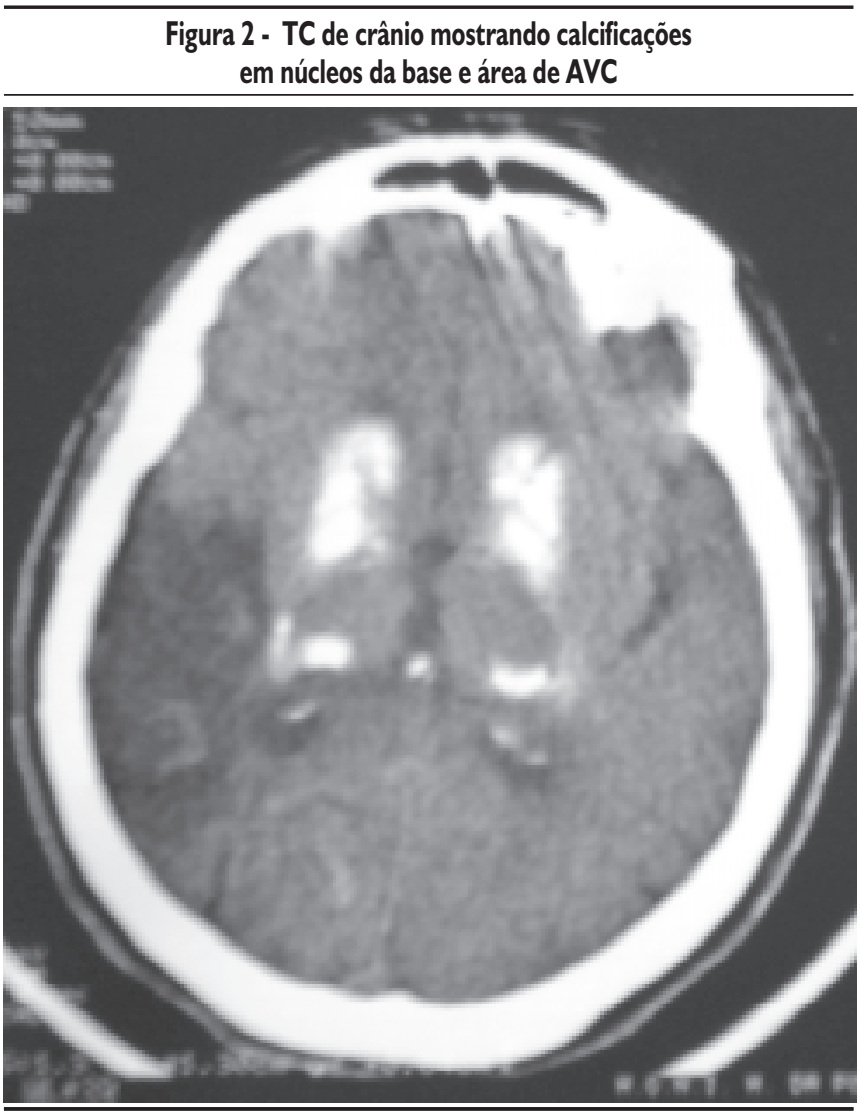




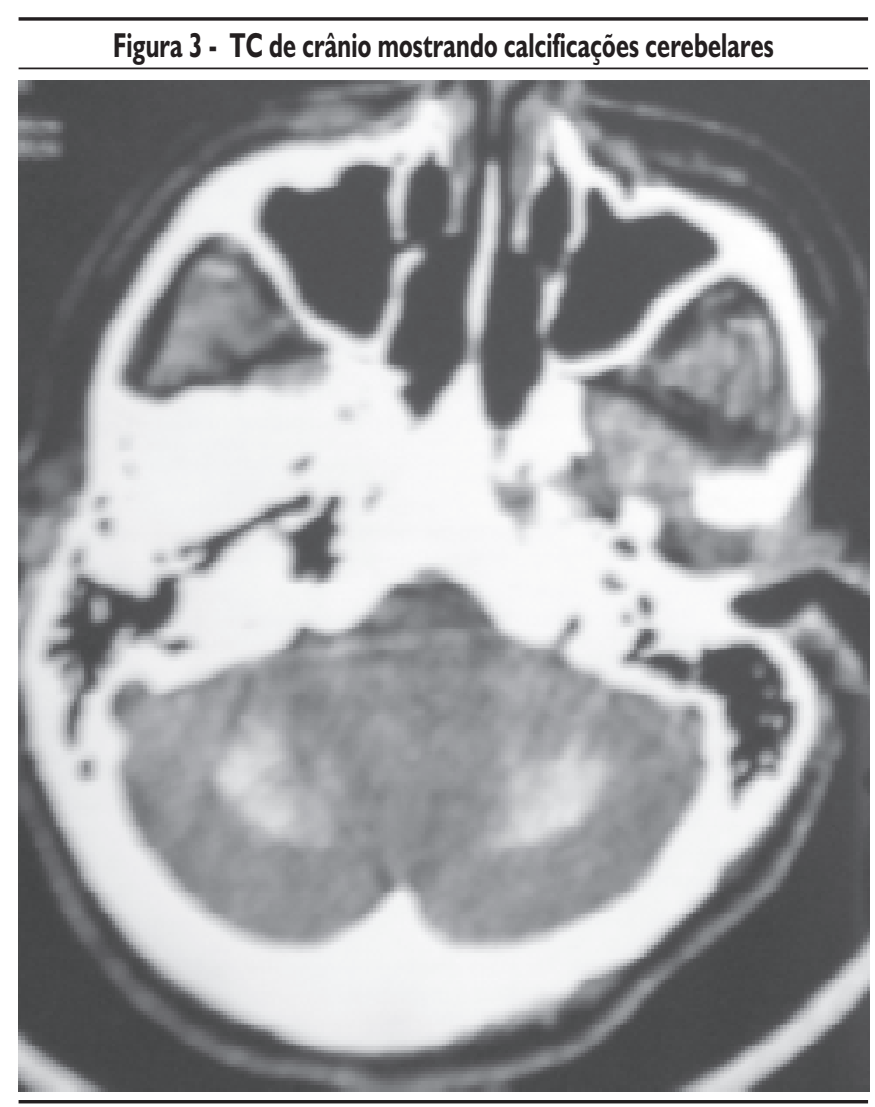

observados por calcificações extensas envolvendo diferentes regiões do encéfalo como núcleos da base, núcleo denteado, tronco cerebral e regiões periventriculares', 2.

Exame clínico-neurológico cuidadoso e investigação laboratorial apropriada devem ser realizados quando nos depararmos com calcificações cerebrais, para que sejam estabelecidos diagnósticos diferenciais e propostas terapêuticas, pricipalmente quando tais calcificações forem observadas em paciente com idade inferior a 50 anos $^{1,4}$. O diagnóstico e tratamento precoces do hipoparatireoidismo podem minimizar as complicações neurológicas ${ }^{5}$. Tais complicações envolvem manifestações clínicas como parkinsonismo, distonias, convulsões, síndrome cerebelar, sintomas psiquiátricos e deterioração mental, entre outros ${ }^{6,7}$. Apesar de ser possível a ocorrência de transtornos cognitivos em função das calcificações cerebrais ${ }^{7}$, acreditamos que as alterações encontradas no caso em questão tenham sido causadas ou agravadas, principalmente pela lesão isquêmica, pelo fato de tais queixas não existirem antes do evento vascular. A apraxia é uma alteração que está conceitualmente ligada a lesões no hemisfério esquerdo, no entanto alguns estudos destacam que existe participação do hemisfério cerebral direito no desenvolvimento de conceitos motores e sua lesão poderia causar alterações apráxicas ${ }^{8}$, como no caso relatado.

\section{Referências}

I. Piñol-Ripoll, G., et al., Differential diagnosis of intracranial calcifications. Rev Neurol, 2005. 4I(3): I5I- I 55.

2. Baptista, M.V., J. Vale, and O. Leitão, Striato-pallido-dentate calcifications. Acta Med Port. , 1997. 10(8-9): 563-567.

3. João, C.G., et al., Cerebral calcifications due to hypoparathyroidism: considerations about cases diagnosed many years after partial thyroidectomy. Arq Bras Endocrinol Metabol, 2006. 50(6): I33-I I37.

4. Rasmussen, M.J., L. Pilø, and H.R. Nielsen, Basal ganglia calcifications demonstrated by $\mathrm{CT}$. Is further investigation necessary when this is found incidentally?]. Ugeskr Laeger (Abstract), | 99|. | 53(29): 205 |-2053.

5. Stelmasiak, Z., et al., Idiopathic hypoparathyroidism with intracranial calcifications and dominant skin manifestations. Med Sci Monit., 2000. 6(I): I45- 150.

6. Mancini, F., et al., Secondary cervical dystonia in iatrogenic hypoparathyroidism associated with extensive brain calcifications. Functional Neurology, 2006. 2I(3): I65-167.

7. Kowdley, K.V., B.M. Coull, and E.S. Orwoll, Cognitive impairment and intracranial calcification in chronic hypoparathyroidism. Am J Med Sci, 1999. 317: 273-277

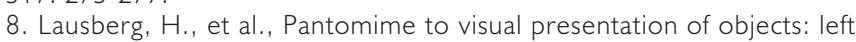
hand dyspraxia in patients with complete callosotomy. Brain, 2003. 126: p. 343-360. 